\begin{abstract}
¿Cómo citar este artículo?
Gallego Quiceno, D. E., Bustamante Penagos, L. E., Quintero Arango, L. F., Jiménez Sánchez, J. I. y

Echeverri Gutiérrez, C. A. (septiembre-diciembre, 2018). La importancia de los recursos

financieros personales y su relación con la inteligencia financiera: revisión documental. Revista Virtual Universidad Católica del Norte, (55), 173 - 191.
\end{abstract}

\title{
| La importancia de los recursos financieros personales y su relación con la inteligencia financiera: revisión documental ${ }^{1}$
}

The importance of personal financial resources and their relationship with financial intelligence: documentary review

\section{Dany Esteban Gallego Quiceno}

Corporación Universitaria Americana

dgallego@coruniamericana.edu.co

\section{Luis Fernando Quintero Arango}

Universidad Católica Luis Amigó

luis.quinterora@amigo.edu.co

\author{
Laura Estefany Bustamante Penagos \\ Corporación Universitaria Americana \\ lbustamante@coruniamericana.edu.co
}

\section{Jorge Iván Jiménez Sánchez}

Universidad Católica Luis Amigó

jorge.jimenezsa@amigo.edu.co

\section{Camilo Andrés Echeverri Gutiérrez}

Corporación Universitaria Americana

cecheverri@coruniamericana.edu.co

Recibido: 05 de septiembre de 2018

Evaluado: 22 de septiembre de 2018

Aprobado: 22 de noviembre de 2018

\section{| Resumen}

El presente artículo muestra los resultados de una investigación sobre la construcción teórica, con respecto a los recursos financieros personales y la inteligencia financiera; el objetivo es analizar, desde una perspectiva teórica, cómo las personas perciben el significado de los recursos financieros, y cómo se relaciona con la inteligencia financiera. La búsqueda documental, se realizó por medio de un análisis sobre los tipos de inteligencia, y la manera en que éstos influyen en la toma de decisiones financieras. El rastreo bibliográfico permitió la construcción de cinco categorías de análisis, relacionadas con los recursos financieros, a saber: diagnóstico de educación financiera, competencias para la toma de decisiones financieras, capacidad de generación de ingresos, percepción de inseguridad y estrés financiero. En los hallazgos de la revisión teórica, se encontró que las personas relacionan recursos financieros con el salario; la teoría explica, que los excedentes financieros es una forma en que se pueden realizar inversiones, pero factores de gastos inesperados y no tener cultura de ahorro, afectan comprender la inteligencia financiera. Finalmente, se concluye que las personas consideran como, si administran los recursos financieros, se pueden generar ingresos adicionales, a partir de ahorros que esta o el grupo familiar realizan.

Palabras clave: Comportamiento financiero, Inteligencia financiera, Recursos financieros, Toma de decisiones.

1 Artículo de revisión, derivado del proyecto de investigación: El manejo de los recursos económicos y la inteligencia financiera de los estudiantes de educación superior, de la Facultad de Ciencias Económicas, Administrativas y Contables FCEAC, cofinanciado entre la Universidad Católica Luis Amigó y la Corporación Universitaria Americana. 


\section{| Abstract}

The present article shows the results of an investigation on the theoretical construction, with respect to the personal financial resources and the financial intelligence; The objective is to analyze, from a theoretical perspective, how people perceive the meaning of financial resources, and how it relates to financial intelligence. The documentary search was carried out through an analysis of the types of intelligence, and the way in which they influence financial decision-making. The bibliographic tracking allowed the construction of five categories of analysis, related to financial resources, namely: diagnosis of financial education, skills for financial decision making, income generation capacity, perception of insecurity and financial stress. In the findings of the theoretical review, it was found that people relate financial resources to salary; the theory explains, that the financial surplus is a way in which investments can be made, but factors of unexpected expenses and not having savings culture, affect understanding financial intelligence. Finally, it is concluded that people consider how, the financial resources are administered, additional income can be generated, based on savings that the person or the family group makes.development of the competencies of the students who are close to completing the undergraduate academic programs. The purpose of this document is to analyze the convergences and divergences of the program content of the Civil Engineering program of the Militar University of New Granada -UMNG- in the areas of engineering sciences and applied engineering, with respect to the Saber Pro Testing guidelines. The methodology used is based on documentary research, as information is organized in matrices to perform an analysis and interpretation of it, and thus establish the relationship between research categories. Finally, and as a conclusion, the convergence in the development of most of the competences formulated is validated; for the divergences some strategies will be proposed.

Keywords: Financial behavior, Financial intelligence, Financial resources, Decision making.

\section{| Introducción}

La presente revisión documental busca explicar cómo la administración de los recursos financieros tiene repercusiones, y la forma en que las personas administran sus ingresos; además, considera un análisis sobre la inteligencia financiera, de acuerdo al comportamiento económico de las personas. Desde la propuesta teórica de Gardner (1983) sobre las inteligencias múltiples, se abre un análisis, entre lo que tiene relación con la cultura financiera y su vínculo con la inteligencia financiera, de importancia hoy para los países, la economía, el grupo familiar y las personas.

Por tanto, surge un marco metodológico que soporta los conceptos relacionados con la administración de los recursos financieros económicos y la inteligencia financiera. Zárate y Matviuk (2010) proponen una relación directa entre las emociones de las personas, y cómo la misma está ligada a la inteligencia financiera que se tiene en cuenta al momento de realizar inversiones en portafolios financieros; así mismo, Aguirre (2015) define que la inteligencia estratégica está relacionada con los recursos de las personas y la forma en que se puede abordar la inteligencia financiera, haciendo así un proceso de vinculación entre los recursos financieros de las personas, la estrategia de cómo invertir y la forma en dónde y en qué invertir. 
Siguiendo lo que expresa Galeano (2015), el propósito de este estado del conocimiento es el de: "recuperar sistemática y reflexivamente el conocimiento acumulado sobre un objeto o tema central de estudio" (p. 141). En esta órbita, luego de realizar el rastreo, se seleccionaron teóricos principales, tales como: Gardner (1983 y 1999), Garay (2016), Bray (2002) y Sternberg (1981, 1985 y 2003), los cuales permitieron dar cuenta de unas categorías emergentes en la materia de análisis. Los rastreos se realizaron frente a los conceptos de recursos financieros y la inteligencia financiera; adicional, se estableció la relación con las competencias financieras de las personas, sus repercusiones individuales y colectivas.

\section{| Metodología}

Para la construcción de este artículo de revisión teórica, se tomó el concepto de inteligencia desde la perspectiva de la investigación, y la relación dialógica y compleja entre los recursos financieros y la inteligencia financiera. Igualmente, se establecieron unas categorías emergentes como: diagnóstico de educación financiera, competencias para la toma de decisiones financieras, capacidad de generación de ingresos, percepción de inseguridad y estrés financiero, y capacidad de evaluación de riesgos y oportunidades financieras. Para el desarrollo de los temas antes expuestos, se hizo un análisis de artículos derivados de investigación y de revisión teórica, por medio de la búsqueda en bases de datos especializadas, como: Redalyc, Dialnet, Ebsco, Scopus y Google Académico; luego de procesar la información, se realizó un filtro desde los conceptos que tenían relación directa con los temas: recursos financieros e inteligencia financiera, descritos en el título y en las categorías emergentes; así mismo, se contempló considerar los autores más representativos, entre ellos algunos clásicos, sobre los temas propuestos en este trabajo.

Así las cosas, el artículo tiene una revisión teórica acerca de la cultura financiera y la inteligencia financiera, como dos categorías principales, en aras de articular otros temas o categorías emergentes que permitan estructurar la revisión bibliográfica sobre el tema propuesto, que cobra hoy importancia, en contextos sociales, familiares, académicos, económicos, culturales y en el desarrollo de la misma sociedad, con el fin de mejorar las condiciones de vida.

\section{Marco teórico}

\section{Concepto de inteligencia desde la perspectiva de la investigación}

En lo que respecta a la inteligencia, la investigación se apoya en los conceptos desarrollados por Gardner (1983), autor de la teoría de las inteligencias múltiples. Para el autor no existe un concepto único u homogenizante del significado de la inteligencia, sino que tiene que ver con el desarrollo de competencias del sujeto para adaptarse y evolucionar:

(...) hasta ahora la palabra inteligencia se ha limitado básicamente a las capacidades lingüisticas y lógicas, aunque el ser humano puede procesar elementos tan diversos como los contenidos del espacio, la música o la psique propia y ajena. Al igual que una tira elástica, las concepciones de la inteligencia deben dar aún más de sí para abarcar estos contenidos tan diversos. (Gardner, 1999, p. 202). 
Con este autor, se expande el paradigma de lo que se ha entendido por inteligencia, exponiendo dicho significado a la relación no sólo lógica y lingüística, sino de diferentes esferas humanas, que antes no se entendían como inherentes a la misma. Para Gardner (1999), el paradigma objetivista, que negaba a la inteligencia su relación con otras esferas de lo humano, ha decaido, es por ello que la inteligencia se entiende como una circunstancia que tiene que ver con la época, la sociedad y la cultura, de manera que no existe una sola forma de entenderla. Hay, en consecuencia, dos cuestiones principales de la inteligencia, en referencia a la teoría de Gardner (1999); en principio, la imposibilidad de describirla de manera general, sin importar el tiempo, la cultura o la historia del individuo; y segundo, la imposibilidad de entenderla en el presente, de manera unidimensional; la inteligencia en términos complejos interrelaciona las habilidades humanas, de manera que la misma se expone como un conjunto de competencias o habilidades que tienen éxito tanto en la expresión teórica como en la práctica, a partir de una relación de equilibrio, en vez del volumen, eminentemente lógico o lingüístico.

Para Gardner, aunque existen varias condiciones de la inteligencia, la principal es su adecuación al medio social: "Una inteligencia humana debe ser genuinamente útil e importante, al menos en determinados ambientes culturales" (Gardner, 1983, p. 96). Por ello, reformula la definición de inteligencia como: “Un potencial bio-psicológico para procesar información que se puede activar en un marco cultural para resolver problemas o crear productos que tienen valor para una cultura" (Gardner, 1999, p. 45).

Con esta nueva definición se da ruptura al componente lógico en exclusiva, y se adhieren las necesidades psicológicas y su importancia sociocultural. Por ello, se trazan unos criterios bio-psicológicos para catalogar una inteligencia; a saber: a) alternativa que una persona sea separada por una lesión cerebral; b) personas que muestren un comportamiento diferente, frente a la media de la población; c) existencia de mecanismos básicos de transformación de la información, implicados en ella; d) historia de desarrollo evolutivo específico, identificable en términos ontogenéticos; e) historia de desarrollo evolutivo específico, en términos filogenéticos; f) apoyo de los hallazgos procedentes de la psicología experimental; g) apoyo de las aportaciones de la tradición psicométrica; y h) posibilidad de codificación en un sistema simbólico (Gardner, 1983).

Y, finalmente, Gardner (1983) considera que el ser humano debe tener unas necesidades psicológicas, y que tiene un impacto en el contexto social y cultural. Desde la inteligencia, con respecto a otros seres vivientes, el ser humano expresa lo simbólico, el significado y la creación de una simbología, así como la forma en que se interpretan los resultados de las experiencias vividas en su larga vida, recordando razonamientos coherentes y claros frente a los símbolos.

Según lo anterior, se amplía el horizonte tradicional y se da vía para las evaluaciones de esta área, en relación con factores antes olvidados de la experiencia humana, y relegados a un carácter inferior a los razonamientos lógico-lingüísticos.

Ahora bien, otro autor que expande el marco del conocimiento frente al tema de la inteligencia es Sternberg (1981) con la teoría triárquica, en la cual diferencia tres dimensiones básicas de la inteligencia, tal y como lo presentan Mora y Martín (2007): “el contexto (en el que aparece), la experiencia (que se tiene al respecto) y los componentes (que la integran). La 
Teoría Triárquica (1985) queda definida por tres subteorías, una por cada una de las dimensiones propuestas" (p. 81). Así las cosas, la inteligencia se definirá como la: "actividad mental dirigida hacia la adaptación intencionada, selección y formación de medios del mundo real relevantes para la vida de uno" (Sternberg, 1985, p. 45).

Sobre la dimensión contextual, se enfatiza en la importancia del ambiente, del momento de la vida y de los sujetos, definiendo así una mayor subjetividad en la valoración de la inteligencia, en razón a que: "lo que se requiere para la adaptación, selección y configuración puede variar según las personas o grupos de personas, así como según los medios o ambientes" (Sternberg, 1985, p. 46); por lo cual existirán distinciones, dependiendo de las personas, de sus momentos de vida y de la comunidad a la cual pertenezca el individuo.

Lo anterior, afianza una visión de la inteligencia como interrelación compleja que se realiza en el ser humano y que interactúa con sus circunstancias específicas, sin que pueda definirse de manera globalizante para la totalidad de los de su especie; en consecuencia, la inteligencia: "no es simplemente la habilidad de aprender y razonar sobre nuevos conceptos, sino la habilidad de aprender y razonar sobre nuevos tipos de conceptos" (Sternberg, 1981, p. 4). De manera que la inteligencia no es una propiedad innata, sino un factor que se desarrolla con el tiempo, el cual evoluciona y se transforma con un sinnúmero de condiciones que circundan la praxis cotidiana del sujeto y de la sociedad en general.

En razón de lo dicho, la inteligencia evoluciona, cambia, se adapta, tanto para la vivencia del individuo como para su actuar en la sociedad. Es la facultad de como la inteligencia genera cambios en la conducta de las personas, en la transformación y cambios de la información, a través de la capacidad ejecutiva de adaptarse y adquirir nuevos conocimientos, los cuales permitan los cambios necesarios para que se logre la sistematización de la información (Sternberg, 1985).

Los dos autores en análisis componen una comunicación clara y distinta, porque aceptan una formación contextual y variable de la inteligencia humana: “Coincidiendo con Sternberg (1985), la propuesta de Gardner $(1983,1999)$ reconoce la relatividad de la inteligencia, consecuencia de su contextualización, a la vez que se apoya en el criterio funcional para proclamar su universalidad" (Mora y Martín, 2007, p. 71). La inteligencia no podrá ser explicada ya por factores condicionantes de la biología, sino que tendrá que entenderse su carácter transformacional, dependiendo del contexto de formación, y con base en las respuestas cotidianas del sujeto a los problemas lógicos y sociales que encuentra en su vida comunitaria. Lo anterior, dará como resultado la existencia de mayores o menores posibilidades creadas por el sujeto, para su adaptabilidad en el entorno y la mejora de su propia subsistencia.

La capacidad de adaptación al entorno y en especial al contexto de decisiones financieras, requiere de destrezas, tanto para lograr el éxito en la vida, en términos de posibilidad, así como en el ámbito social y cultural. La habilidad que se tenga para alcanzar buenos resultados se logra a través de concretar las fortalezas y trabajar en robustecer las debilidades. Lograr un equilibrio entre fortalezas y debilidades, depende de la capacidad que tenga el individuo para poder buscar la adaptación a los diferentes contextos; los buenos resultados se pueden lograr a través de la capacidad de encontrar los entornos apropiados y desarrollar la creatividad, el análisis y los juicios con rigor; asi se puede contemplar que la inteligencia financiera requiere de aspectos externos, pero lo principal es lo interno, lo que puede controlar el individuo 
(Sternberg, 2003).

Teniendo en cuenta lo antes descrito, es fundamental entender que la inteligencia no es un terreno que aplica exclusivamente para la comprensión, la lógica, los aspectos memorísticos o lingüísticos, sino que se acerca a la adaptabilidad del sujeto al entorno y a las especificidades que le exige la vida comunitaria. Así las cosas, debe reconocerse que, en el presente, una de las habilidades contextuales de mayor importancia hace referencia a la inteligencia, desde un punto de vista financiero. En un entorno en el que las transacciones globales son la apuesta y en el que el libre flujo del mercado implica la asimilación de unas economías emergentes, es ineludible la administración inteligente y la adaptación a las condiciones que exige el mercado.

De acuerdo con lo anterior, Garay (2016) expresa que los usuarios identifican el sistema financiero, pero no tienen conocimiento acerca de cómo cada persona puede desarrollar una inteligencia financiera, acorde a las necesidades del mercado y de sus situaciones particulares; como los expresa Stemberg (1985) el contexto, la experiencia y el componente, hacen parte fundamental para comprender una inteligencia, no solo desde la racionalidad del ser humano, sino desde la inteligencia financiera y el administración de los recursos.

Así las cosas, el sistema financiero colombiano es una oportunidad que está a la mano, y sus diferentes opciones, en cuanto a las modalidades de crédito y financiación, son una oportunidad; la inteligencia financiera, entonces, está presente al momento de tomar la decisión de contratar un crédito, en tanto hay que tener en cuenta la capacidad de pago y los futuros compromisos, para poder cumplirlos. La banca debe empezar a ofrecer nuevos productos financieros, con el fin de mejorar la calidad de vida de las personas; bien es sabido que la banca comercial no presta fácil, y por ello se deben incorporar nuevos productos financieros que favorezcan a las personas que más lo necesitan (Jiménez, Rojas y Ramírez, 2017). En este orden de ideas, es importante que la banca cuente con nuevos productos que mejoren, entre ellos, el financiamiento exclusivo de la educación superior, y para llevar a cabo proyectos de emprendimiento de baja inversión, pero que sean registrados de forma legal (Jiménez, 2014).

\section{Relación dialógica y compleja entre los recursos financieros y la inteligencia financiera}

El bienestar financiero hace parte de una de las circunstancias necesarias de la vida, en el tiempo que corre, y por tal, la inteligencia contextualizada debería responder de manera eficiente y asertiva a estos mandatos de la praxis social; este concepto engloba tres aspectos ineludibles: "I) características individuales, II) comportamientos financieros y III) acontecimientos financieros estresantes. Por lo tanto, el sector laboral económicamente activo se ve enfrentado a diferentes situaciones derivadas de la adquisición de deudas y sus responsabilidades" (Kim, Garman, \& Sorhaindo, 2003, como se citó en Vallejo Trujillo, Vázquez García y Martínez Rangel, 2016, p. 39).

De otro lado, se puede relacionar el bienestar financiero con la inteligencia financiera, en tanto estos dos conceptos se encuentran. Aun cuando la inteligencia financiera es la clave para obtener el bienestar financiero individual, la misma debe contemplar las necesidades 
colectivas, en razón a que el bienestar financiero del sujeto, a largo plazo, depende de su capacidad, de la inteligencia financiera y la evolución de la comunidad que lo contiene.

Tal y como afirman Vallejo Trujillo et al. (2016) la sociedad en conjunto depende de la educación financiera del individuo y de su accionar como parte de la colectividad:

(...) es importante anotar que el bienestar financiero o la situación financiera, son el reflejo del nivel de educación financiera que cada quien posee, el cual, impacta en el progreso social el crecimiento económico y los indicadores sociales, así como en los indicadores del desarrollo del sistema financiero de un país. (pp. 37-38).

Así, surge la pregunta por la existencia de la inteligencia contextual, que permite llegar a ese concepto clave para la construcción del sujeto y de la colectividad; esto es, el bienestar financiero. Así las cosas, se requiere saber ¿cómo se logra este estado intelectivo y ético del sujeto, en el que él proporciona y prevé los medios para su bienestar financiero? Ante esta pregunta, la respuesta clave es: a partir de la educación financiera, la cual debe entenderse, según Organisation for Economic Co-operation and Development Staff -OECD- (2005), como el proceso por medio del cual las personas adquieren una mejor comprensión de los conceptos, productos y servicios financieros, cuyo propósito es desarrollar las habilidades necesarias para tomar decisiones informadas y evaluar tanto riesgos como oportunidades financieras, en aras de mejorar su bienestar. Siguiendo con este tema, Fuenzalida y Ruíz (2009) se refieren a la incapacidad en la generación de ingresos, lo que puede causar en el individuo angustia de tipo financiero. Lo anterior, es comentado por Mills, Grasmick, Stout \& Wenk (1992, como se citó en Vallejo Trujillo y Martínez Rangel, 2016) al asegurar que:

(...) la incapacidad en la generación de ingresos, puede o no causar en el individuo, estrés o angustia financiera, aunque no siempre es posible generalizar que dos personas con el mismo nivel de ingreso presenten el mismo nivel de estrés o bienestar financiero. (p. 84).

Sin embargo, Duarte Cáceres, Rosado Muñoz y Basulto Triay (2014) consideran que sí hay afectación al bienestar, porque: "cuando las personas experimentan problemas financieros (como tener medios inadecuados para cumplir con las demandas financieras de la familia) su estrés financiero incrementa y su bienestar sufre" (p. 136). Se entiende que, ante la imposibilidad de recursos para el cumplimiento de las deudas contraídas, surge la angustia financiera: "se dice que está en situación de angustia financiera cuando existe la posibilidad (aunque sea leve o lejana) de que no pueda hacer frente a sus compromisos" (Guillén Romo, 2009, p. 18). Pero, esta angustia del sujeto, como se dijo en anterior aparte, es sólo un efecto inmediato de la falta de asertividad de la inteligencia contextual, más específicamente de la financiera, debido a que a largo plazo la sociedad en conjunto es la que se ve afectada, y, por tanto, el individuo vuelve a sentir menoscabado su bienestar.

Esta situación que evidencia una afectación al bienestar financiero particular y global, es afianzada cuando Montoya (2005) y Olin (2014) dan cuenta de las repercusiones sociales del daño individual y empresarial que genera el mal manejo del dinero. Situaciones que se presentan en el contexto financiero pueden afectar a las personas y a las empresas; de hecho, el excesivo gasto, el endeudamiento con créditos en diferentes entidades financieras, los gastos 
realizados sin fundamentos equilibrados y sin análisis, según las capacidades de pago, y un flujo de caja negativo son factores que influyen en mantener unas finanzas sanas y enfocadas en generar utilidad (Montoya, 2005). Estos factores no influyen en el detrimento particular exclusivamente, sino que tienen un efecto en las organizaciones, que puede derivar en la pérdida de productividad, liquidez financiera, y colaboradores insatisfechos con su labor y los salarios recibidos. En algunas investigaciones se menciona que los colaborados que tienen una remuneración salarial justa son aquellos que tienen una inteligencia financiera exitosa, y los empleados con remuneraciones bajas, tienen un comportamiento financiero deficiente (Montoya, 2005).

En ese mismo sentido, Olin (2014) considera que el mundo actual exige cada vez más la comprensión ética del ciudadano, frente a su papel en el entorno globalizado: "es significativo pensar que se vive en un mundo globalizado y, que exige la buena administración de nuestras finanzas personales, lo que requiere conocimientos sobre el funcionamiento del sistema financiero" (p. 15).

Por ello, la base de este tipo de educación financiera consiste en hacer entender al ciudadano tanto la importancia que tiene en su vida como las implicaciones comunitarias que sus acciones tienen en el desarrollo de su época y entorno; para Rivera Ochoa y Bernal Domínguez (2018), considerar la educación financiera, es identificar cómo están definidos los productos financieros, y conocer la mejor opción para la toma de decisiones, de manera que se permita encontrar un equilibrio que tenga impacto en la calidad de vida, y se cumpla con los objetivos y metas financieras propuestas; además, las alternativas financieras con las que cuenta un mercado, permiten diversificar el portafolio de inversión y dan cuenta de un balance eficiente de los ingresos y gastos, o en algún momento en las pérdidas y ganancias de las diferentes inversiones que se realicen (Olin, 2014).

Asimismo, la educación financiera aporta en el conocimiento de las exigencias y consecuencias de un mundo globalizado, en el cual, tal y como afirma Argandoña (1993), no se cuenta con unos parámetros válidos para determinar el consumo, la durabilidad de los productos y servicios, la oferta por parte de las empresas, la inversión en horas que se realiza en el trabajo, los cambios en los estilos de vida, el incremento del estrés por factores asociados al trabajo, a las deudas y la pérdida de afecto, el índice elevado de ansiedad, los problemas psicológicos por ciudades contaminadas y saturadas de personas, vehículos generando afectación en el medio ambiente y la carencia de valores y el respeto por los demás; los anteriores elementos impactan en que no se cuente con una educación financiera equilibrada, según las necesidades del mercado y de las personas.

De manera que la educación financiera se puede ver afectada por los factores antes mencionados, pero los individuos deben fortalecerse para afrontar esas vicisitudes, propendiendo por la consecución de un bienestar general que equilibre las necesidades del entorno y también la calidad de vida del individuo. Argandoña (1993) considera otros factores asociados a la educación financiera y las inversiones, entre ellos: el estrés que produce la seguridad en los rendimientos financieros de un portafolio, el afán de ganar utilidades rápido y tomar decisiones apresuradas, y el corto plazo que se tiene para que una inversión se recupere de una situación negativa que suceda en el mercado; los anteriores son elementos determinantes para que la calidad de vida personal se vea afectada y tenga impacto en sus integrantes, influyendo en las relaciones humanas y personales. 
Este equilibrio entre los factores que componen la calidad de vida y las necesidades financieras, son analizadas por el Foro Mundial de Educación (2000), enfatizando en la educación para el desarrollo económico social y humano. En esta línea, la Organización de las Naciones Unidas para la Educación, la Ciencia y la Cultura -Unesco- (2000) considera que el éxito financiero y económico de una inversión, tiene que ver con lo competentes que sean las personas. Algunas culturas tienen una educación financiera avanzada, con respecto a otras; por eso, el éxito de esas economías desarrolladas y de las personas que cuentan con una cultura de ahorro e inversión tienen una capacidad de análisis mejor fundamentada, con respecto a economías de nivel medio o bajo.

De otro lado, la educación financiera propenderá por la competencia equilibrada de los individuos; para la Unesco (2000) el desarrollo de un país y de su economía tiene relación con la educación, y lo financiero está dentro de esa cultura de ahorro desde temprana edad, logrando consolidar en la etapa adulta la importancia que tiene establecer inversiones en diferentes portafolios; de hecho, no crear cultura de ahorro y preparación para inversiones de largo plazo, hace que se genere un efecto directo en la poca preparación de los jóvenes y se distorsione el crecimiento económico de una nación, ya que el asistencialismo va directo a sostener a los menos favorecidos, por ende los menos preparados educativamente.

Así mismo, es indispensable que, en el futuro, se fortalezca la educación financiera; Olin (2014) propone que la inteligencia financiera, es un factor adaptativo, que determina la sobrevivencia de las sociedades y de la especie. Esta educación financiera, en un mundo globalizado, debe constituirse en un plan de primer orden, ya que se considera la educación como un derecho humano fundamental, clave en el desarrollo de las naciones, de una inmejorable calidad de vida, y una forma de pertenecer a los sistemas sociales y económicos importantes del mundo, para aportar al desarrollo equilibrado de las diferentes economías.

La educación financiera permitirá que cada uno de los individuos, como engranajes del Estado, contribuyan al fortalecimiento colectivo, en aras de lograr un equilibrio entre los países que han sufrido inequidades, mucho más cuando se está ante el panorama de un capitalismo de avanzada que requiere sujetos educados financieramente y en uso de sus inteligencias contextuales, con el fin de evitar la dominación y la inequidad; además, los efectos del capitalismo global ejercen una presión alta para los países, las economías y las personas (Garay, 2016).

Por otra parte, en la medida en que los mercados se expanden, el poder social y jurídico se ve limitado por las grandes potencias. En este contexto, la educación financiera se constituye en el principal medio para la lucha contra cualquier forma de imposición y de inequidad. Puede decirse, entonces, que el mundo actual exige a la inteligencia nuevas lecturas, y que en el acertado desarrollo financiero del individuo se libran luchas sociales y colectivas. Lo anterior, puede ser causa para disminuir la brecha de equidad educativa financiera, que permita adquirir un conocimiento sobre la cultura educativa, la cual repercute en mejorar la calidad de vida; es así como: "Las políticas de descentralización educativa se han fundamentado como las más apropiadas para remover una mirada de causas de problemas estructurales presentes en la enseñanza básica: acceso, retención, egreso, pertinencia, participación, eficiencia, innovación, aprendizaje y equidad" (Helvia Rivas, 1992; Pardo, 1999; Winkler \& Gershberg, 2000, como se citó en Fernández Aguerre, 2010, p. 237). 
Lo complejo de articular un diálogo coherente entre recursos financieros y la inteligencia financiera es que, en los países de mediano y bajo desarrollo, la población débil, como son mujeres, niños y personas de la tercera edad, deben primero considerar sus necesidades básicas, y sí consiguen tener excedentes, lograr una inversión pequeña y a corto tiempo, contrario a lo que se presenta con economías desarrolladas, que consideran la educación financiera como una necesidad de desarrollo y de cambio; así mismo, las necesidades básicas son satisfechas de manera rápida, logrando excedentes que permiten realizar inversiones constantes y con períodos más largos (Cohen y Franco, 2006, como se citó en Fernández Aguerre, 2010).

Así, Aguirre (2015) asevera que lo cognitivo de las personas hace que se tenga una sociedad de análisis y no de impulsos, de mirada a largo plazo y no a corto plazo; así mismo, una mejor toma de decisiones permite fortalecer las inteligencias múltiples, considerando que esto es un avance relevante para la sociedad y las economías de los países. Por ello, desde los países en vía de desarrollo, se deben crear políticas públicas que incentiven el ahorro, la cultura financiera y la educación financiera.

Para la Unesco (2000), vincular principios, valores y un equilibrio en el desarrollo sostenible, aplicado desde la educación, impulsará cambios en el comportamiento de las personas, en aras de mejorar la calidad de vida actual y futura, considerando que el desarrollo sostenible y sustentable también se logra con la cultura financiera, el ahorro y la inversión, lo cual permita articular lo humano, pero también lo sostenible en el tiempo.

Se afianzan en esta definición los conceptos de desarrollo sostenible y bienestar financiero equilibrado, que permitan, según Unesco (2006), solucionar problemas de impacto social, que se busquen alternativas de vida sin impactar en el equilibrio de los recursos naturales y no intervengan en la equidad social e igualdad de su entorno, de manera especial los mismos de su especie.

Dicha capacidad de analizar problemas, que repercuta en decisiones asertivas, requiere competencias que le faciliten la administración eficiente de los bienes y la toma de decisiones financieras inteligentes, tal y como lo afirman Cohen, McGuinness, Sebstad y Stack (2005). Una de estas competencias corresponde a la administración del riesgo, el cual: "es considerado como un proceso interactivo e iterativo, basado en el conocimiento, la evaluación y la medición de los riesgos y sus impactos, cuyo propósito fundamental sea el de mejorar la toma de decisiones en las organizaciones" (García Santillán, Herrera Santiago y Edel Navarro, 2006, p. 28).

De otro lado, se requiere adquirir competencias financieras que faciliten la toma de decisiones, y que, además, permitan una educación financiera apropiada, considerando el análisis de factores de tipo macroeconómico, microeconómico, geopolítica, sociología y cultura, así como de aspectos propios de cada país y región, con el fin de realizar inversiones donde el riesgo sea mínimo (Sánchez Parrales, Suriaga Sánchez y Bonilla Freire, 2016).

El estudio de estos factores permitirá tomar decisiones que repercutan en el beneficio particular: "Las finanzas tienen muchos aspectos y elementos relacionados con los procesos de administración y demás aspectos que inciden en las decisiones de las personas para poder invertir el dinero en negocios que tengan rentabilidad" (Sánchez Parrales et al., 2016, p. 14). 
Ahora bien, los proyectos de inteligencia de negocios propenden por buscar inversiones con altos rendimientos, pero con pocos desequilibrios; según lo anterior, se considera importante el apoyo de expertos de análisis de comportamientos futuros de inversiones, donde la tecnología, con el apoyo de software especializados, que modelan escenarios futuros, permita conocer posibles situaciones en los mercados, que son volátiles y cambiantes de manera vertiginosa (Sánchez Parrales et al., 2016). En consecuencia, se hace fundamental la educación financiera para propender por la inteligencia, el bienestar financiero particular y los proyectos de inteligencia de negocios en las empresas.

En suma, la inteligencia financiera permite prever los comportamientos financieros del individuo y evitar los acontecimientos financieros estresantes (Bray, 2002). Para Duarte Cáceres et al. (2014): "Ayudar a cambiar el comportamiento financiero proporciona un enfoque integral para mejorar tanto el bienestar financiero como la salud" (p. 137). Este cambio de comportamiento tiene que ver con el autoconocimiento y la educación, "la educación incrementa la habilidad de los individuos para adquirir y procesar información para la práctica de comportamientos financieros positivos" (Duarte Cáceres, et al., 2014, p. 132).

Lo anterior, no solo repercute en la estabilidad del individuo y de la sociedad, sino en el aumento de sus finanzas; para un individuo y la misma sociedad, contar con ingresos y considerar una alta calidad de vida, se considera tranquilidad y una riqueza relativa; esto hace que las personas sientan placer por su actividad laboral, y directamente se incremente la productividad y competitividad de las organizaciones, del país y de la sociedad. Los ingresos, la renta y otros ingresos derivados de inversiones, hace que se tenga una calidad de vida inmejorable, esto relacionado con menos enfermedad, estrés y preocupaciones por su situación financiera; lo anterior, relaciona un estado ideal que tienen los países desarrollados y las personas que cuentan con una cultura e inteligencia financiera (Duarte Cáceres et al., 2014, p. 136).

En esta medida, avanza el individuo y la comunidad a la cual pertenece. Es así como la inteligencia financiera y la cultura financiera forman parte de la estructura económica, social, cultural y religiosa de un país. Garay (2016) considera que sí los individuos tienen una capacidad de ahorro, por medio de una cultura programada del mismo, pueden generar riqueza, impactando la productividad y permitiendo que, a futuro, se tenga oportunidad de mejorar la calidad de vida y el crecimiento de la esperanza de la misma.

\section{Resultados}

Después de valorar los temas y autores que permitieron formar una conceptualización del área, puede afirmarse que son claras unas líneas categoriales emergentes en la interrelación de los autores y el desarrollo de los conceptos. Las categorías se sintetizarán a continuación: Bray (2002) argumenta lo relacionado con la educación financiera; luego, Cohen et al. (2005) dedican su teoría a considerar la eficiencia de los bienes financieros y la toma de decisiones; seguido, aparecen Fuenzalida y Ruíz (2009) haciendo énfasis en la incapacidad del individuo para 
generar ingresos; y finalmente, se exponen las teorías del estrés financiero y percepción de inseguridad en las inversiones, atribuidas a Argandoña (1993) y Bray (2002).

Desde las lógicas de las categorías emergentes propuestas, se contempla como cada autor asume una posición argumentativa, crítica o no, frente al tema de la inteligencia financiera y los recursos financieros. Contextualizando cada una de las líneas emergentes, se considera apropiado observar, de forma integral, la educación financiera, como la forma en que se piensa y se realiza alguna inversión, con el fin de convertirla en una alternativa rentable, y no asegurando que siempre será así, en tanto esto permitirá la toma de decisiones acertadas; nadie pondría en riesgo su capital en una inversión que no genere rentabilidad. Así mismo, los individuos y el grupo familiar deben de considerar la generación de otros ingresos adicionales a su salario, ya que ello permitirá construir un fondo común de ahorro, para realizar inversiones según las alternativas y opciones del mercado y de los inversionistas.

En este párrafo, y no menos importante, se encuentra lo que hace referencia al estrés financiero y a la percepción de inseguridad que se tiene al momento de realizar una inversión. En la actualidad, los cambios presentados en la geopolítica y la economía global, la turbulencia en los negocios, y las decisiones políticas y económicas, de los llamados países potencia, se consideran como un riesgo de generación de incertidumbre para los inversionistas, en tanto las decisiones que estos países tomen, logran tener un impacto directo en las condiciones macroeconómicas de todo el mundo.

\section{Líneas categoriales emergentes}

Las categorías de análisis que se proponen están relacionadas con el recurso financiero de las personas y la inteligencia financiera, la cual se puede lograr con el fin de distribuir esos recursos. Las categorías emergentes seleccionadas, a partir de la revisión teórica, es decir, educación financiera, competencias para la toma de decisiones financieras, capacidad de ingresos y estrés financiero, hacen un compendió, en aras de concretar las categorías que se pretende desarrollar en el trabajo de campo, que será la otra fase de la investigación propuesta.

En la línea de las categorías emergentes, existe un interés actual por identificar cómo se puede establecer, dentro de la sociedad, las familias y cada individuo, una educación financiera adecuada, que permita consolidar unos niveles de inversión altos, con el fin de mejorar la calidad de vida. En este sentido, los núcleos familiares pueden realizar un presupuesto familiar, que logre evidenciar un diagnóstico del estado actual de sus finanzas, para luego crear estrategias que permitan tomar decisiones, con respecto a realizar inversiones o no.

\section{Diagnóstico de educación financiera}

La educación financiera hace parte tanto de las nuevas perspectivas sobre la inteligencia, su descripción, desarrollo y evolución, como de las necesidades comunitarias en un mundo globalizado. El diagnóstico de la educación financiera permitirá determinar el grado de desarrollo de un ciudadano y de una sociedad, y las posibilidades que la misma tiene para lograr adaptarse al movimiento económico mundial, y, con ello, contrarrestar las posibles inequidades que trae consigo el desconocimiento de las exigencias financieras y la deficiente capacitación 
para la educación de la inteligencia financiera. Garay (2016) analiza que la educación financiera está ligada a la calidad de la educación y al contexto en que está desarrollada; es decir, influyen aspectos como la cultura, la sociedad y la educación, que se haya recibida desde joven, para poder tener una idea de lo que es realizar un diagnóstico financiero, con el fin de ejecutar determinadas inversiones en los diferentes sectores económicos que ofrece el mercado.

De otro lado, la educación financiera debe ser considerara por los países y el sistema educativo de cada país, iniciando desde la básica, como una manera de crear en los individuos estrategias de cultura de inversión desde temprana edad; esto llevará a que los indicadores de calidad de vida, economía y sociedad mejoren, y se pueda identificar que el efecto del ahorro sí tiene un impacto positivo en toda la sociedad y sus diferentes escenarios.

\section{Competencias para la toma de decisiones financieras}

Las competencias en este sentido pueden adquirirse con el aprendizaje, pero el proceso de adquisición inicial tiene qué ver con el desarrollo del sujeto, la comunidad en la que se desarrolla y su adaptabilidad con el entorno. Aun así, entendiendo que con los autores valorados se esclarece la idea de que la inteligencia no es un concepto estático ni que puede aplicarse de manera homogénea, siempre está latente la posibilidad de adquirir conciencia sobre las necesidades financieras propias y del entorno, y de desarrollar competencias para afrontar, de manera equilibrada, tanto las finanzas personales como la relación con el sistema mundial, mediante acciones que posibiliten el crecimiento y el desarrollo sostenible. Bray (2002) analiza que la toma de decisiones financieras se expresa desde la empresa, la sociedad y la familia; prepararse para poder lograr un equilibrio al momento de tomar decisiones financieras es fundamental en una sociedad; no solo es el gasto, el ahorro es importante para poder tener posibilidades de un mejor futuro.

Así mismo, Cohen et al. (2005) destacan que la educación financiera debe estar soportada en decisiones financieras fundamentadas, según necesidades de cada persona; es claro que cada individuo tiene una forma de pensar al momento de invertir su dinero, es por eso que aparecen diferentes tipos de inversionistas que, según la clasificación y tipo de inversión, tienen aspiraciones y pretenden recibir algo más a cambio, y considerar la mejor opción que exista en el mercado; las personas son prudentes y su riesgo casi siempre es moderado, esto es algo común dentro de la cultura colombiana, que quiere invertir poco pero ganar alta rentabilidad.

\section{Capacidad de generación de ingresos}

La capacidad para generar ingresos se relaciona con la inteligencia financiera y con la evaluación del riesgo; asimismo, con las capacidades del sujeto para hacer parte del engranaje social. Esta capacidad de generar ingresos repercute en el bienestar financiero del sujeto, fortaleciendo o no sus relaciones con el entorno; y también, repercute a largo plazo con el desarrollo de la sociedad y la posibilidad de equidad dentro de la misma. La angustia financiera por incapacidad de generación de ingresos repercute en las demás esferas del sujeto y afecta la forma de vida de éste. Así mismo, se desplaza hacia las necesidades sociales, generando inseguridad en el sistema y crisis en la credibilidad financiera colectiva. 
Duarte Cáceres et al. (2014) destacan que los recursos financieros, sumado a la inteligencia financiera, pueden llevar al resultado de tener una capacidad de ingresos que no se tenía, partiendo de analizar un tipo de inversiones que puede ser prudente y recomendado para las personas; el generar ingresos adicionales, es impactar la calidad de vida y mejorar aspectos de satisfacción personal.

La capacidad de un individuo para generar nuevos ingresos está determinada por el potencial de crecimiento propio, soportada en no afectar sus ingresos principales y poder crear estrategias que le permitan consolidar sus actividades; a partir de esto, se considera que el individuo puede lograr un ingreso adicional, el cual siendo bien manejado puede tener repercusiones positivas para él y su grupo familiar. En otro ámbito, la generación de nuevos ingresos puede fundar repercusiones en el contexto social y económico, como lo pueden ser el estrés financiero e incertidumbre en la inversión de cualquier tipo.

De otro lado, el tema de la generación de ingresos es una invitación para el sector financiero, en tanto son ellos los llamados a la captación de esos excedentes, con el fin de consolidar el proceso de captación y colocación de dinero o de otro tipo de inversiones, pero entregando una rentabilidad apropiada, para estimular el ahorro y la inversión; esto se logra, como antes se expresó, incentivando la cultura financiera desde pequeños, e iniciando estudios básicos en el sistema educativo.

\section{Percepción de inseguridad y estrés financiero}

El estrés financiero ocurre por la falta de un uso equilibrado de la inteligencia contextual, debido a que el sujeto no logra adaptarse a los cambios del entorno y afrontar asertivamente los requerimientos de la sociedad, para obtener bienestar financiero. Este estrés impone una carga al sujeto y afecta la percepción y seguridad de las demás actividades vitales del mismo, pero, además, influye en la inseguridad creciente del sistema; afecta las posibilidades de equidad, en un mundo en el cual el dominio del poder económico es creciente; y limita las posibilidades de desarrollar las competencias para la educación financiera.

Argandoña (1993) establece que las personas, al recibir su dinero por una labor ejecutada, realizan un balance entre el activo (lo que queda) y el pasivo (lo que debe), y al momento de establecer esa relación y percibir que sus gastos son superiores, genera una insatisfacción financiera, una percepción de saber que trabaja para cubrir sus deudas y que no queda para otras situaciones necesarias de subsistencia; así las cosas, se empieza a sufrir de un estrés financiero, el cual lleva a conductas de insatisfacción personal, con el mismo y su entorno, juzgando su actuar, y pensando en qué otras actividades puede crear para equilibrar su vida financiera.

Por consiguiente, la percepción de insatisfacción financiera también puede desencadenar en otros escenarios, como enfermedades de tipo muscular, cerebrales y cardiológicos, donde la afectación puede ser parcial o total; así mismo, los efectos jurídicos y legales, los cuales se presentan por incumplimientos o pérdida de las inversiones, se contemplan como otro factor de estrés y preocupación al momento de invertir. Teniendo en cuenta lo anterior, el riesgo en una inversión, de cualquier tipo, existe; lo único real es minimizar el riesgo, en tanto evitarlo será imposible. 


\section{Capacidad de evaluación de riesgos y oportunidades financieras}

En la actualidad, y a partir del desarrollo de los estudios sobre la posibilidad intelectiva humana, se entiende que es necesario comprender no solo la actividad lógica coherente o lingüística del sujeto, sino la interacción de éste con su entorno y la toma de decisiones asertivas para su propia evolución. En el mismo sentido, es fundamental para el sujeto entenderse parte del entorno, obrar conforme a la ética de su papel y encontrar caminos seguros de desarrollo, en medio de unas políticas globalizantes que imponen cargas de agilidad, inmediatez e incertidumbre a la praxis humana. Es por ello que la capacidad de evaluación del riesgo y la comprensión de las oportunidades financieras son fundamentales en la aplicación de la inteligencia del sujeto al entorno, y contribuyen a la generación de seguridad financiera global, evolución educativa y bienestar de la colectividad.

Las condiciones de los hogares se encuentran supeditadas a que cada uno de sus integrantes aporte, para contribuir con los gastos de la familia; según Fuenzalida y Ruíz (2009) existen factores para que las familias perciban dificultades al momento de pagar sus deudas; el entorno social y cultural hacen que se identifiquen factores como gastos "hormigas" o innecesarios, que van permeando esas obligaciones. Teniendo en cuenta lo anterior, es necesario analizar los riesgos que se corren al momento de que una persona, del grupo familiar, se quede sin empleo o no cumpla con sus compromisos; además, qué oportunidades financieras se pueden encontrar en el mercado para poder cumplir con sus responsabilidades; analizar temas de endeudamiento, inversión y ahorro entre el grupo familiar soporta momentos de crisis o situaciones difíciles.

De otro lado, las oportunidades financieras están abiertas; se debe analizar la mejor decisión al momento de invertir, pero, evaluar el riesgo puede ser una forma de reducir la pérdida de dinero en alguna inversión. Las crisis financieras no solo se presentan en los hogares, el mundo también ha vivido diferentes momentos, en los cuales el impacto financiero, para algunos países, ha sido crítico y de alto impacto, llevando a todo el sistema productivo, económico, social y financiero a un colapso; no obstante, lo anterior permite identificar lo sucedido y tomar acciones, en aras de mejorar esas condiciones adversas, tanto en los países como en los hogares y el individuo.

\section{Discusión}

El desarrollo de este artículo de revisión teórica, permitió que se lograrán identificar aspectos relacionados con la cultura financiera y la inteligencia financiera. Desde el aporte que se pretende realizar, es necesario considerar los siguientes aspectos: crear estrategias que permitan una mirada a la importancia de la cultura del ahorro, y que las personas y el grupo familiar se acerquen a las lógicas de un ahorro o fondo común, el cual permita establecer aspectos básicos de ingresos y egresos, donde los excedentes logren construir un portafolio de inversión familiar.

En el caso de los excedentes monetarios y considerar inversiones de cualquier tipo, existe una relación directa entre la rentabilidad, el éxito, el fracaso y la incertidumbre, llevando a cabo aspectos de contexto social, económico, político y cultural frente al manejo de esos excedentes financieros, los cuales se pueden lograr en algún momento; así las cosas, contar con otros 
ingresos es una alternativa que puede favorecer el ahorro y mejorar condiciones y calidad de vida.

Las categorías emergentes, miradas cada una desde el contexto, logran dar un aporte a los constructos propuestos, es decir a los recursos financieros y a la inteligencia financiera. La capacidad de inversión, el estrés financiero, educación financiera, competencias para la toma de decisiones financieras, capacidad de ingresos y estrés financiero, hacen un compendió para considerar, de forma holística, que la cultura y toma de decisiones, al momento de realizar una inversión, se debe mirar, considerando diversos aspectos, desde la forma en que un individuo piensa en su estructura mental y la capacidad que tiene para realizar inversiones, hasta la adaptación, al momento de asumir una pérdida o cambios en la rentabilidad que se tenía contemplada.

Desde la aplicación práctica, se propone que las personas realicen sus inversiones en diferentes alternativas que existen en el mercado, pero se deben contemplar los riesgos inherentes y relacionados, al momento que se toma la decisión de invertir; los riesgos no se pueden evitar, se pueden minimizar, por medio de inversiones que se consideren seguras. No obstante, lo que se debe dejar a un lado, es optar por aquellas inversiones que suelen pagar altos intereses o rendimientos en un corto plazo, lo cuál puede ser considerado como inseguro, ya que el mercado financiero tiene un comportamiento similar dentro de las tasas de interés de captación.

De igual manera, dentro del análisis realizado, se encontró la relación que se tiene entre el estrés financiero y la toma de decisiones, es decir, la incertidumbre que existe al momento que una persona desea invertir, esperando una rentabilidad apropiada o la pérdida total o parcial del capital invertido. El estrés financiero puede afectar el rendimiento laboral y el comportamiento de la persona en su entorno social.

\section{| Conclusiones}

En primer lugar, es necesario establecer cómo, desde cada persona, se generan unos recursos, en este caso de tipo financiero, y se estructura la forma en la que se destinan sus ingresos para diversas actividades de la vida cotidiana. Es recomendable que las personas consideren como desde la inteligencia financiera, es decir, la forma eficiente de manejar sus recursos, se puede construir unos ingresos adicionales, a partir de excedentes o ahorros que la persona o el grupo familiar puede realizar.

Dentro de las categorías de análisis expuestas en el desarrollo del trabajo, se llegó a la consideración de que la educación financiera es un elemento fundamental para poder hacer inversiones de diversas índoles; sumado a que la cultura, la sociedad y la formación académica están ligadas a la manera en que se propone esa educación financiera. Así mismo, se puede adicionar la toma de decisiones financieras y las competencias que las personas pueden tener para pensar en realizar inversiones, buscar asesorías o proponer qué inversión se realiza, al poner en la balanza aspectos positivos y negativos de una determinada inversión.

De otro lado, el generar inversiones apropiadas, es recibir otros ingresos; es por eso importante el cómo las personas determinan qué excedentes se esperan recibir sobre una determinada inversión; esto se logra con una destinación de recursos y una inteligencia 
financiera, que permita obtener dinero por un tiempo determinado, conociendo el riesgo en que se puede incurrir; toda inversión siempre tiene un determinado riesgo, sea bajo, moderado $o$ alto.

En el desarrollo de la revisión teórica se evidenció que aún faltan investigaciones empíricas, que aporten a la construcción de una cultura financiera y al desarrollo de una educación financiera, y que permitan conocer cuál es el estado actual, proponiendo algún modelo práctico, en aras de que las familias y las personas logren desarrollar una cultura de inversión y consideren que el ahorro a largo plazo tiene aspectos positivos que permiten mejorar la calidad de vida, la estabilidad financiera y el impacto social, avanzando a un sociedad justa y equitativa.

La debilidad de los hogares y los individuos, frente a la cultura financiera, es un reflejo del desarrollo de los países, es por eso que esta revisión teórica permitió identificar como en Colombia aún falta realizar un trabajo eficiente y estructurado de buscar el desarrollo de los individuos, la familia y la sociedad, por medio de la inversión y el ahorro.

\section{| Referencias}

Aguirre, J. (enero-marzo, 2015). Inteligencia estratégica: un sistema para gestionar la innovación. Revista Estudios Gerenciales, 31(134), 100-110. Recuperado de http://www.re dalyc.org/pdf/212/21233043011.pdf

Argandoña, A. (1993). La ética de la sociedad de consumo. Cuadernos Empresa y Humanismo, (37), 1-32. Recuperado de http://dadun.unav.edu/bitstream/10171/3847/1/Cuaderno037.pdf

Bray, R. (2002). Hardship in Australia: An analysis of financial stress indicators in the 1998-99 Australian Bureau of Statistics Household Expenditure Surevey. Occasional Paper series, (4), 1-100. Recuperado de https://www.dss.gov.au/about-the-department/publica tions-articles/research-publications/occasional-paper-se ries/number-4-hardship-in-australia-an-analysis-of-financ ial-stress-indicators-in-the-1998-99-australian-bureau-of-sta tistics-household-expenditure-survez

Cohen, M., McGuinness, E., Sebstad, J. y Stack, k. (2005). Estudios de mercado de la educación financiera. Washington, Estados Unidos: Microfinance opportunities. Recuperado de https://oicolombia.com.co/upload/edu_financiera/Conceptos_fi nancieros/Estudio_de_mercado_-_documento_de_trabajo_no_2.pdf

Duarte Cáceres, L. G., Rosado Muñoz, Y. L. y Basulto Triay, J. H. (julio-diciembre, 2014). Comportamiento y Bienestar Financiero como factores competitivos en el personal académico de una Institución de Educación Superior. Mercados y Negocios, 15(2), 129-148.

Fernández Aguerre, T. (2010). La desigualdad de aprendizajes, 1995-2006. En F. Cortés y O. De Oliveira (Coords.), Problemas de México (pp. 235-262). México: El Colegio de México. 
Fuenzalida, M. y Ruíz, J. (agosto, 2009). Riesgo financiero en los hogares. Economía Chilena, 12(2), 35-53. Recuperado de https://www.researchgate.net/publication/28321757_Riesgo_ Financiero_de_los_Hogares

Galeano, M. E. (2015). Estrategias de investigación social cualitativa: el giro de la mirada. Medellín, Colombia: La Carreta Editores.

Garay, G. (mayo, 2016). Índice de alfabetismo financiero, la cultura y la educación financiera. Revista Perspectivas, (37), 23-40. Recuperado de http://www.redalyc.org/articulo.oa?i $d=425946304003$

García Santillán, A., Herrera Santiago, G. y Edel Navarro, R. (marzo, 2006). Decisiones Financie ras y la Administración del Riesgo desde el enfoque del aprendizaje (Un acercamiento teórico a una propuesta metodológica). Contribuciones a la Economía. Recuperado de http://www.eumed.net/ce/2006/ags-0603.htm?iframe=true\&width=95\%\&height=95\%

Gardner, H. (1983). Frames of Mind. The Theory of Multiple Intelligences. Nueva York, United States: Basic Books.

Gardner, H. (1999). Intelligence Reframed: Multiple Intelligences for the 21st Century. Nueva York, United States: Basic Books.

Guillén Romo, H. (2009). Kindleberger y Galbraith: el eterno retorno de las crisis financieras. Mundo Siglo XXI, (18), 15-25. Recuperado de http://132.248.9.34/hevila/Mundosi gloXXI/2009/no18/2.pdf

Jiménez, J. (2014). Nuevas modalidades de financiación para microempresas. Puente Revista Científica, 8(2), 1-12. Recuperado de https://revistas.upb.edu.co/index.php/puente/arti cle/view/7132

Jiménez, J., Rojas, F. y Ramírez, D. (2017). Situación del Crowdfunding como mecanismo para la financiación de Pymes en Colombia. Espacios, 38(43). Recuperado de http://www.revist aespacios.com/a17v38n43/17384324.html

Montoya, P. (2005). Educación Financiera. Estudio aplicado a: Comisión Federl de Electricidad. Zona metropolitana poniente (Tesina de maestría). Universidad Autónoma de Nuevo León. Monterrey, México.

Mora, J. A. y Martín, M. L. (2007). La concepción de la inteligencia en los planteamientos de Gardner (1983) y Sternberg (1985) como desarrollos teóricos precursores de la noción de inteligencia emocional. Revista de Historia de la Psicología, 28(4), 67-92.

Olin, B. J. (2014). La educación financiera como base para la toma de decisiones personales de inversión (Tesis de maestría). Universidad Autónoma de Querétaro. Santiago de Querétaro, México.

Organisation for Economic Co-operation and Development Staff-OECD-. (2005). OECD 
factbook 2005: economic, environmental and social statistics. Paris, France: OECD.

Organización de las Naciones Unidas para la Educación, la Ciencia y la Cultura -Unesco-. (2000). Informe Final. Foro Mundial sobre la Educación. París, Francia: Unesco. Recupera do de http://unesdoc.unesco.org/images/0012/001211/121117s.pdf

Organización de las Naciones Unidas para la Educación, la Ciencia y la Cultura -Unesco-.

(2006). Decenio de las Naciones Unidas de la Educación con miras al Desarrollo Sostenible (2005-2014): Plan de aplicación internacional. París, Francia: Unesco. Recupera do de http://unesdoc.unesco.org/images/0014/001486/148654so.pdf

Rivera Ochoa, B. E. y Bernal Domínguez, D. (mayo, 2018). La importancia de la educación financiera en la toma de decisiones de endeudamiento. Estudio de una sucursal de "Mi Banco" en México. Revista Perspectivas, 21(41), 117-144.

Sánchez Parrales, L. A., Suriaga Sánchez, M. A. y Bonilla Freire, J. D. (marzo, 2016). Finanzas en los negocios inteligentes. Revista Caribeña de Ciencias Sociales. Recuperado de http://www.eumed.net/rev/caribe/2016/03/finanzas.html

Sternberg, R.J. (1981). Intelligence and Nonentrenchment. Journal of Educational Psychology, 73(1), 1-16. Recuperate de http://psycnet.apa.org/record/1981-06882-001

Sternberg, R. J. (1985). Beyond IQ: A triarchic theory of human intelligence. Cambridge, United Kingdom: Cambridge University Press.

Sternberg, R. J. (2003). Construct validity of the theory of successful intelligence. En R. J. Sternberg, J. Lautrey \& T. I. Lubart (Eds.), Models of intelligence. International perspectives (pp. 55-80). Washington, United States: American Psychological Association.

Vallejo Trujillo, L. S y Martínez Rangel, M. G. (enero-junio, 2016). Perfil de Bienestar Financiero: aporte hacia la mejora de una comunidad inteligente. Investigación Administrativa, (117), 82-95. Recuperado de: http://www.redalyc.org/pdf/4560/456046142006.pdf

Vallejo Trujillo, L. S., Vázquez García, A. W. y Martínez Rangel, M. G. (julio-diciembre, 2016). Bienestar financiero, una reflexión desde la ficción neoliberal en un contexto local. In Vestigium Ire, 10(2), 32-47. Recuperado de http://revistas.ustatunja.edu.co/index.php/i vestigium/article/view/1270

Zárate, R. y Matviuk, S. (julio-diciembre, 2010). La inteligencia emocional y el sector financiero colombiano. Revista Escuela de Administración de Negocios, (69), 148 - 165. Recuperado de http://www.redalyc.org/pdf/206/20619966009.pdf 